\title{
Forkhead Box Protein C2 (FOXC2) Promotes the Resistance of Human Ovarian Cancer Cells to Cisplatin In Vitro and In Vivo
}

\author{
Chanjuan Li Hongjuan Ding Jing Tian Lili Wu Yun Wang Yuan Xing Min Chen
}

Department of Obstetrics and Gynecology, Nanjing Maternity and Child Health Care Hospital to Nanjing Medical University, Nanjing, P.R. China

\section{Key Words}

Forkhead box protein C2 $•$ Cisplatin • Chemoresistance • Ovarian cancer

\begin{abstract}
Background/Aims: FOXC2 has been reported to play a role in tumor progression, but the correlations of FOXC2 with the cisplatin (CDDP) resistance of ovarian cancer cells are still unclear. The purpose of the present study is to investigate the roles of FOXC2 in the CDDP resistance of ovarian cancer cells and its possible mechanisms. Methods: Quantitative real-time PCR (qRT$P C R$ ) was performed to detect the expression of FOXC2 mRNA in CDDP-resistant or sensitive ovarian cancer tissues and cell lines (SKOV3/CDDP and SKOV3). Gain- and loss-of-function assays were performed to analyze the effects of FOXC2 knockdown or overexpression on the in vitro and in vivo sensitivity of ovarian cancer cells to CDDP and its possible molecular mechanisms. Results: The relative expression level of FOXC2 mRNA in CDDP-resistant ovarian cancer tissues was higher than that in CDDP-sensitive tissues. Also, the expression of FOXC2 mRNA and protein in CDDP-resistant ovarian cancer cell line (SKOV3/CDDP) cell line was higher than that in its parental cell line (SOKV3). Small hairpin RNA (shRNA)-mediated FOXC2 knockdown significantly increased the in vitro and in vive sensitivity of SKOV3/CDDP cells to CDDP by enhancing apoptosis, while upregulation of FOXC2 significantly decreased the in vitro and in vivo sensitivity of SKOV3 cells to CDDP by reducing apoptosis. Furthermore, FOXC2 activates the Akt and MAPK signaling pathways, and then induced the decreased expression of Bcl-2 protein and the increased expression of Bax and cleaved caspase- 3 proteins. Conclusions: FOXC2 mediates the CDDP resistance of ovarian cancer cells by activation of the Akt and MAPK signaling pathways, and may be a potential novel therapeutic target for overcoming CDDP resistance in human ovarian cancer.
\end{abstract}

\section{Introduction}

Ovarian cancer accounts for approximately $3 \%$ of all female cancers around the world [1]. The carcinogenesis of ovarian cancer is a multi-step and complicated process, which 


\section{Cellular Physiology Cell Physiol Biochem 2016;39:242-252

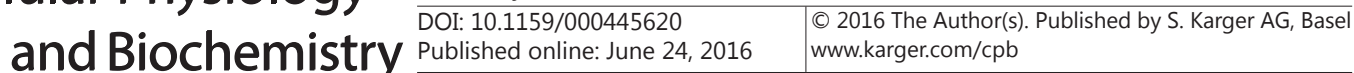 \\ Li et al.: FOXC2 and CDDP Resistance of Ovarian Cancer Cells}

involves activation of oncogenes and inactivation of tumor suppressor genes [2-5]. Despite advances in surgery and adjuvant therapy, the 5-year survival rate of patients with ovarian cancer is not still improved during the past few decades. For patients with advanced ovarian cancer, the current standard treatment involves primary cytoreductive surgery followed by a platinum agent (cisplatin or carboplatin) - based chemotherapy. However, a sizable proportion of the patients show tumor recurrence after chemotherapy which is mainly caused by the development of drug resistance [6]. Therefore, it is necessary to elucidate the molecular mechanisms involved in tumor chemoresistance will contribute to developing a promising strategy for the treatment of chemoresistant ovarian cancer.

Forkhead box protein $\mathrm{C} 2$ (FOXC2; also known as mesenchyme forkhead 1) belongs to Forkhead box (Fox) transcription factors family [7]. The overexpression of FOXC2 has been found to play important roles in regulation of tumor angiogenesis and development [8]. It has been reported that FOXC2 plays a key role in metastasis and is associated with aggressive basal-like breast cancers [9]. Also, haplodeficiency of FOXC2 could lead to impaired formation of tumor blood vessels as well as reduced tumor growth, suggesting that FOXC2 is critical for tumor development and angiogenesis. Meanwhile, high expression of FOXC2 is reported to be an independent prognostic factor in glioma, esophageal cancer, gastric cancer and non-small cell lung cancer [10-13]. Recently, overexpressed FOXC2 is found to enhance the epithelial-to-mesenchymal transition and invasion of ovarian cancer cells [14]. However, the correlation of FOXC2 expression with the sensitivity of ovarian cancer cells to CDDP is unclear and remains to be further elucidated. In the present study, we will detect the expression of FOXC2 in CDDP-sensitive or resistant ovarian cancer tissues and cell lines. Then, gain-and loss-of-function assays were performed to investigate the roles of FOXC2 in the CDDP resistance of ovarian cancer cells and its underlying molecular mechanisms.

\section{Materials and Methods}

\section{Cell culture}

Human CDDP-resistant and parental ovarian cancer cell lines (SKOV3/CDDP and SKOV3) were purchased from Xinyu Biotechnology Co. Ltd (Shanghai, China). All cell lines were cultured in RPMI 1640 (GIBCO-BRL) medium supplemented with $10 \%$ fetal bovine serum (FBS), $100 \mathrm{U} / \mathrm{ml}$ penicillin, and $100 \mu \mathrm{g} /$ $\mathrm{ml}$ streptomycin in humidified air at $37^{\circ} \mathrm{C}$ with $5 \% \mathrm{CO2}$. CDDP were purchased from Sigma-Aldrich (USA).

\section{Tissue samples}

The study has been performed with the approval of the Ethics Committee of the Jiangsu Province Medical Association. Ovarian epithelial cancer tissues were obtained by needle biopsy from 20 patients with advanced ovarian cancer who received chemotherapy at Jiangsu Hospital from May 2009 and July 2011. Patients met all of the following criteria: patients who suffered from primary ovarian cancer; a histological diagnosis of ovarian cancer with at least one measurable lesion; a clinical stage of IV; first-line chemotherapy with paclitaxel $\left(100 \mathrm{mg} / \mathrm{m}^{2}\right.$, i.v. d1) and cisplatin $\left(20 \mathrm{mg} / \mathrm{m}^{2}\right.$, i.v. d1-4) administered every 3 weeks for a maximum of 4 cycles. Tumor response was examined by computed tomography and evaluated according to the Response Evaluation Criteria in Solid tumors (RECIST) as complete response (CR), partial response (PR), stable disease (SD), or progressive disease (PD). The chemotherapy-sensitive subjects were defined as the total of those achieved CR, PR or SD responses. Tissue samples were snap-frozen in liquid nitrogen, which were transferred to $500 \mu \mathrm{l}$ TRIzol solution immediately after harvesting in order to avoid mRNA degradation. Informed consent was obtained from each patient before tissues sample collection.

\section{Stable transfection}

To ectopically express FOXC2, the plasmid vector (pMD/FOXC2) expressing open-reading frame of FOXC2 was purchased from Sino Biological Inc (Beijing, China). Short hairpin RNA (shRNA) oligonucleotides targeting FOXC2 (shFOXC2, 5'-CCACACGTTTGCAACCCAA-3') and a negative control oligonucleotide (shcontrol, 5'-ACGTGACACGTTCGGAGAA-3') were subcloned into pSilencer4.1-CMVneo vector and the recombinant plasmids were named $\mathrm{pS} / \mathrm{shFOXC2}$ and $\mathrm{pS} /$ shcontrol, respectively. Transfection was 


\section{Cellular Physiology Cell Physiol Biochem 2016;39:242-252 \\ \begin{tabular}{l|l|l} 
and BOI: 10.1159/000445620 & $\begin{array}{l}\text { C } 2016 \text { The Author(s). Published by S. Karger AG, Basel } \\
\text { www.karger.com/cpb }\end{array}$
\end{tabular} \\ Li et al.: FOXC2 and CDDP Resistance of Ovarian Cancer Cells}

performed using Lipofectamine TM 2000 (Invitrogen, USA) according to the manufacturer's instruction. At $48 \mathrm{~h}$ post-transfection, G418 $(800 \mu \mathrm{g} / \mathrm{ml})$ was added to select stable transfectants and individual clones were maintained in a medium containing G418 $(150 \mu \mathrm{g} / \mathrm{ml})$. The stably transfected cells were named SKOV3/CDPP/shFOXC2 (or SKOV3/CDDP/shcontrol) and SKOV3/FOXC2 (or SKOV3/control), respectively.

Quantitative real-time PCR ( $q R T-P C R)$ assay

Total RNA from ovarian cancer tissues or cell lines was isolated using the Trizol reagent (Invitrogen, USA) according to the manufacturer's instruction. Reverse transcript (RT) was carried out with the SuperScript First-Strand Synthesis System for RT-PCR (Invitrogen, USA), and quantitative real-time PCR was carried out using the SYBR Green reporter. The primers used for PCR were as follows: FOXC2 forward 5'-CCTACCTGAGCGAGCAGAAT-3'; reverse 5'-ACCTTGACGAAGCACTCGTT-3'; GAPDH forward 5'-GCACCGTCAAGGCTGAGAAC-3'; reverse 5'-TGGTGAAGACGCCAGTGGA-3'. The data were normalized to the geometric mean of housekeeping gene GAPDH and calculated as $2^{-\triangle \triangle C T}$ method.

Western blot assay

Western blot assay was performed with anti-FOXC2 (Bethyl Laboratories, TX, USA), antiphosphorylated AKT (p-AKT) (Ser473) or phosphorylated ERK 1/2 (p-ERK) (Cell Signaling, CA, USA), antitotal Akt or ERK 1/2, anti-Bcl-2, anti-Bax and anti-cleaved or total caspase-3 (Santa Cruze Biotechnology, CA, USA) as described previously [15]. Anti-GAPDH monoclonal antibody (Santa Cruze Biotechnology, CA, USA) was used as an internal control.

In vitro chemotherapy assay

The single-cell suspensions were prepared and dispersed in 96-well plates. After incubation for 48 $\mathrm{h}$ with the CDDP compounds (Sigma, MO, USA), the $0.5 \mathrm{mg} / \mathrm{mL}$ of MTT solution was added. Following incubation for $4 \mathrm{~h}$, the medium was discarded and $150 \mu \mathrm{L} /$ well of dimethyl sulfoxide (Sigma-Aldrich) was added. The absorbance was measured at $490 \mathrm{~nm}$ using a microplate reader.

Flow cytometric detection of apoptosis

The stably transfected cells were collected for detection of apoptosis using the Annexin V-fluorescein isothiocyanate (FITC) / Propidium Iodide (PI) Apoptosis Detection Kit (BD Pharmingen, USA) according to the manufacturer's instructions. All of the samples were assayed in triplicate.

In vivo chemotherapy assay

The stably transfected cells (SKOV3/CDDP/shFOXC2 or SKOV3/CDDP/shcontrol, SKOV3/FOXC2 or SKOV3/control) were collected and suspended in $100 \mu \mathrm{L} \mathrm{PBS,} \mathrm{and} \mathrm{then} \mathrm{injected} \mathrm{subcutaneously} \mathrm{into} \mathrm{the}$ right side of the posterior flank of BALB/c athymic nude mice ( $n=10 /$ group). The tumor volume formed was calculated by the following formula: Volume $=\left(\right.$ Length $\times$ width $\left.^{2}\right) \times 0.5$. When the average tumor size reached about $50 \mathrm{~mm}^{3}$, CDDP was given through intraperitoneal injection with a concentration of $3.0 \mathrm{mg}$ / $\mathrm{kg}$, one dose every other day with 3 doses totally. Tumor volume was monitored for 5 weeks and measured once five days. The study was approved by the Ethics Committee of Nanjing Medical University, and carried out in accordance with approved guidelines.

\section{Statistical analysis}

All statistical analyses were performed using the SPSS 17.0 statistical software. Experimental data were expressed as the mean \pm SD of at least three independent experiments. Statistical analyses were carried out using one-way ANOVA and Student's $t$ test. Differences between groups were considered significant at $P<0.05$.

\section{Results}

FOXC2 is significantly upregulated in cisplatin-resistant ovarian cancer tissues or cell lines To investigate the association of FOXC2 expression with cisplatin resistance of ovarian cancer, qRT-PCR assay was performed to detect the expression of FOXC2 mRNA in cisplatin- 


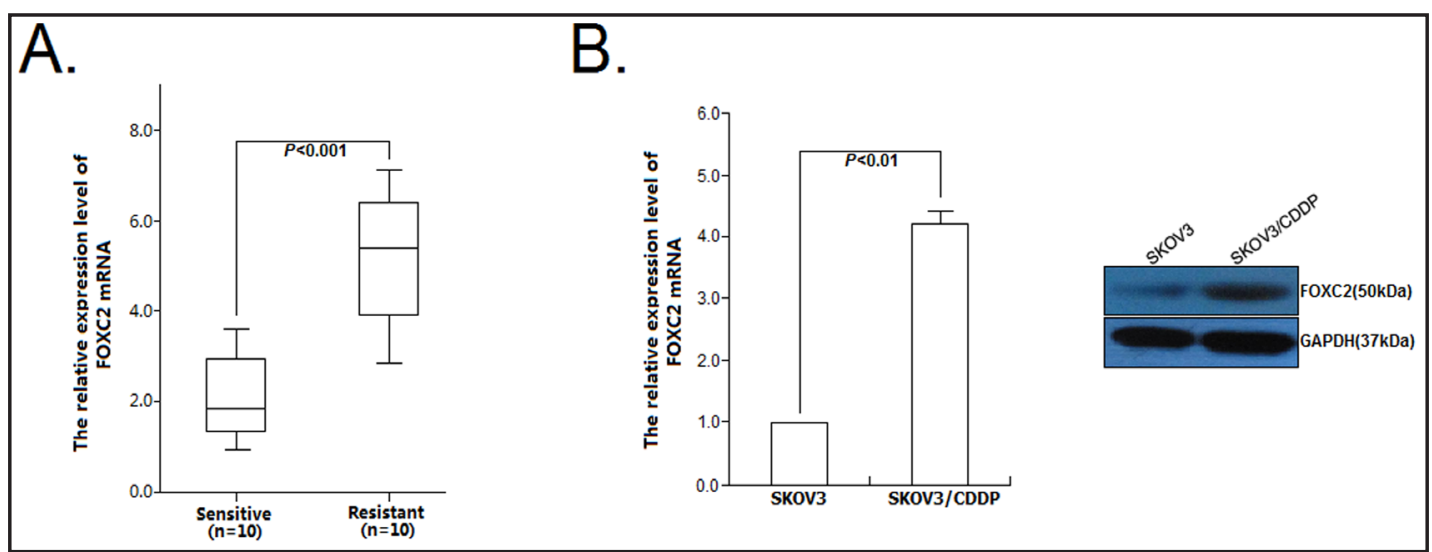

Fig. 1. Correlation between FOXC2 expression and CDDP resistance in ovarian cancer. (A) qRT-PCR detect the relative expression of FOXC2 mRNA in CDDP-resistant ovarian cancer tissues $(n=10)$ and CDDP-sensitive ovarian cancer tissues $(n=10)$. (B) qRT-PCR and Western blot detection of FOXC2 mRNA and protein expression in CDDP-resistant ovarian cancer cell line (SKOV3/CDDP) and its parental ovarian cancer cell line (SKOV3). GAPDH was used as an internal control. All values represent the average of three independent experiments (mean $\pm \mathrm{SD}$ ).

sensitive or resistant ovarian cancer tissues. It was observed that the relative expression level of FOXC2 mRNA in cisplatin-sensitive ovarian cancer tissues $(n=10)$ was significantly lower than those in cisplatin-resistant tissues $(n=10)(P<0.001$; Fig. 1A). Meanwhile, qRTPCR and Western blot assays were performed to detect the expression of FOXC2 mRNA and protein in cisplatin-resistant ovarian cancer cell line (SKOV3/CDDP) and its parental ovarian cancer cell line (SKOV3). As shown in Fig. 1B, the relative expression levels of FOXC2 mRNA and protein in SKOV3/CDDP cell line were significantly higher than that those in SKOV3 cells. These data suggest that upregulation of FOXC2 may play a role in the development of cisplatin resistance in ovarian cancer.

Silencing of FOXC2 increases the chemosensitivity of SKOV3/CDDP cell line to CDDP in vitro and in vivo

To investigate the roles of FOXC2 expression in the CDDP resistance of ovarian cancer cells, the shRNA expression vector targeting FOXC2 (pS/shFOXC2) and the negative control vector (pS/shcontrol) were successfully constructed and stably transfected into SKOV3/CDDP cell line, which was named SKOV3/CDPP/shFOXC2 or SKOV3/CDDP/shcontrol, respectively. qRT-PCR and Western blot assays confirmed the stable knockdown of endogenous FOXC2 in SKOV3/CDPP/shFOXC2 cell line, in comparison with SKOV3/CDDP/shcontrol cell line (Fig. 2A). Then, MTT assay was performed to analyze the effect of FOXC2 knockdown on the $\mathrm{IC}_{50}$ value of CDDP in SKOV3/CDDP cell line. Compared with that in SKOV3/CDDP/shcontrol cells $\left(\mathrm{IC}_{50}: 41.16 \mu \mathrm{M}\right)$, the $\mathrm{IC}_{50}$ value of CDDP in SKOV3/CDDP/shFOXC2 cells $\left(\mathrm{IC}_{50}: 17.23\right.$ $\mu \mathrm{M}$ ) was significantly decreased by approximately $55.7 \%$ (Fig. 2B). When treated with CDDP $(8.5 \mu \mathrm{M})$, the apoptotic rate in SKOV3/CDDP/shFOXC2 cells was significantly increased by approximately $17.80 \%$, in comparison with SKOV3/CDDP/shcontrol cells $(P<0.01$; Fig. 2C). Furthermore, we analyzed the effect of FOXC2 knockdown on the in vivo sensitivity of SKOV3/CDDP cells to CDDP by using nude mice xenograft tumor model. Following CDDP treatments, the tumors formed from SKOV3/CDDP/shcontrol cells grew faster than those formed from SKOV3/CDDP/shFOXC2 cells (Fig. 2D). At 35 days after inoculation, the average volume of tumors formed from SKOV3/CDDP/shFOXC2 cells $\left(456.2 \mathrm{~mm}^{3}\right)$ was significantly smaller than that of tumors formed form SKOV3/CDDP/shcontrol cells $\left(634.6 \mathrm{~mm}^{3}\right)$ $(P<0.001 ;$ Fig. 2E). These data suggest that knockdown of FOXC2 significantly increases the in vitro and in vivo sensitivity of CDDP-resistant ovarian cancer cells to CDDP. 


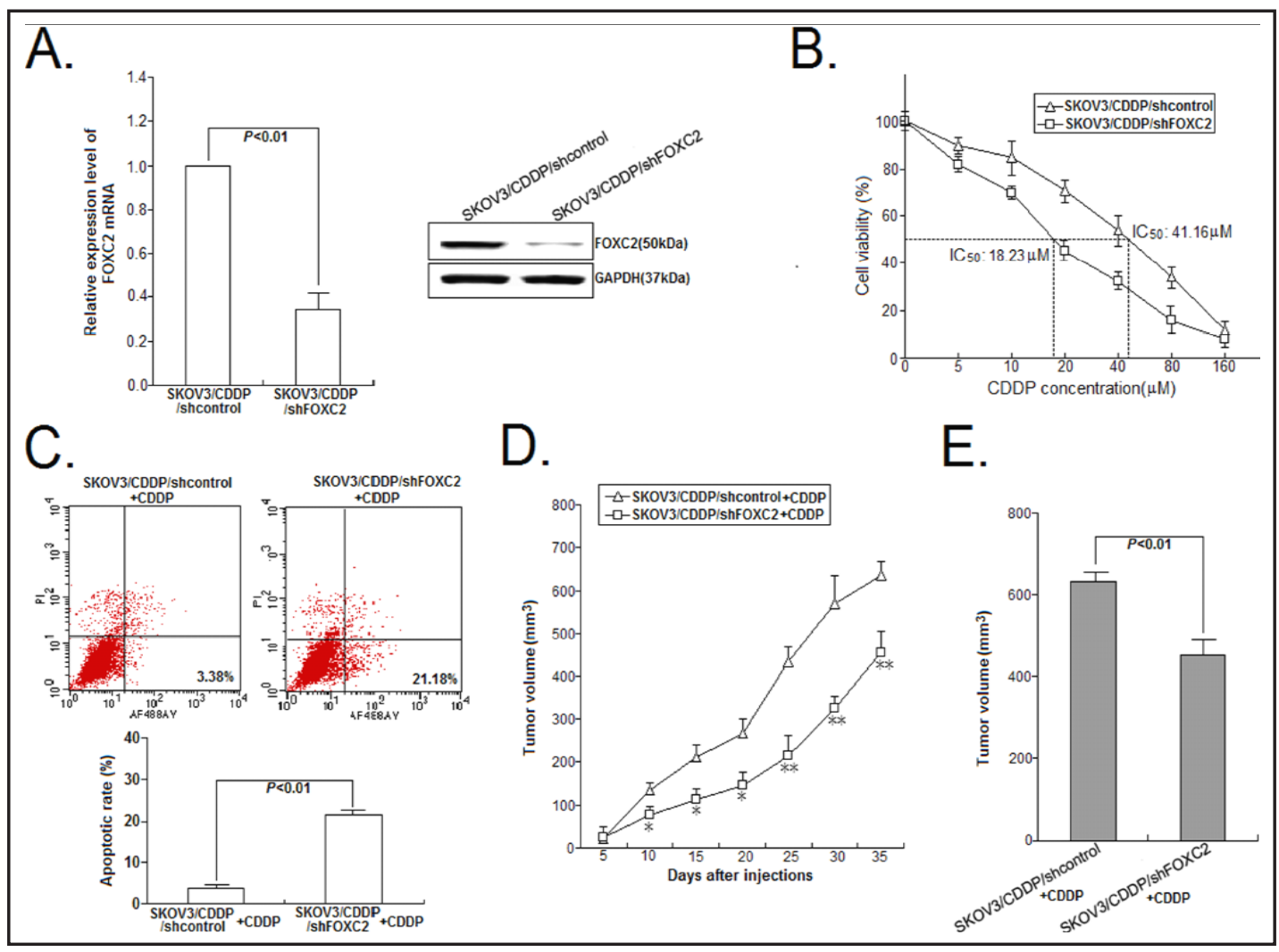

Fig. 2. Knockdown of FOXC2 significantly increases the sensitivity of SKOV3/CDDP cells to CDDP in vitro and in vivo. (A) qRT-PCR and Western blot detection of FOXC2 mRNA and protein expression in SKOV3/ CDDP/shFOXC2 and SKOV3/CDDP/shcontrol cells, respectively. GAPDH serves as an internal control. (B) MTT analysis of the effects of FOXC2 knockdown on the $\mathrm{IC}_{50}$ values of CDDP in SKOV3/CDDP cells. (C) Flow cytometric detection of apoptosis in SKOV3/CDDP/shFOXC2 or SKOV3/CDDP/shcontrol cells with CDDP treatment $(8.5 \mu \mathrm{M})$. (D) The growth in mice of tumors developed from SKOV3/CDDP/shcontrol or SKOV3/ $\mathrm{CDDP} / \mathrm{shFOXC2}$ cells with CDDP treatment. The inoculation was performed in two groups ( $\mathrm{n}=10 /$ group). (E) After 35 days, the average tumor volume of tumors developed from SKOV3/CDDP/shFOXC2 cells with CDDP treatment was significantly smaller than that of tumors developed from SKOV3/CDDP/shcontrol cells with CDDP treatment. All values represent the average of three independent experiments (mean \pm SD). ${ }^{*} P$ $<0.05$ and $* * P<0.01$, compared with the control.

Upregulation of FOXC2 decreases the chemosensitivity of SKOV3 cell line to CDDP in vitro and in vivo

To further investigate the effect of FOXC2 overexpression on the cisplatin sensitivity of ovarian cancer cells, CDDP-sensitive SKOV3 cells were stably transfected with pMD/FOXC2 or $\mathrm{pMD} /$ control, respectively. The results of qRT-PCR and Western blot assays confirmed the upregulation of FOXC2 in SKOV3/FOXC2 cells (Fig. 3A). MTT assay was performed to analyze the effect of FOXC2 upregulation on the $\mathrm{IC}_{50}$ value of CDDP in SKOV3 cell line, and it was observed that the IC ${ }_{50}$ value of CDDP in SKOV3/FOXC2 cells (IC I0 $_{50}: 17.23 \mu \mathrm{M}$ ) was significantly increased by approximately $58.2 \%$, in comparison with that in SKOV3/control cells IC $_{50}$ : $41.16 \mu \mathrm{M})$ (Fig. 3B). When treated with CDDP $(2.5 \mu \mathrm{M})$, the apoptotic rate in SKOV3/ FOXC2 cells was significantly decreased by approximately $10.78 \%$, in comparison with SKOV3/ control cells $(P<0.05$; Fig. 3C). Next, we analyzed the effect of FOXC2 upregulation on the in vivo sensitivity of SKOV3 cells to CDDP. Following CDDP treatments, the tumors formed from SKOV3/FOXC2 cells grew faster than those formed from SKOV3/control cells (Fig. 3D). At 35 days after inoculation, the average volume of tumors formed from SKOV3/FOXC2 cells 


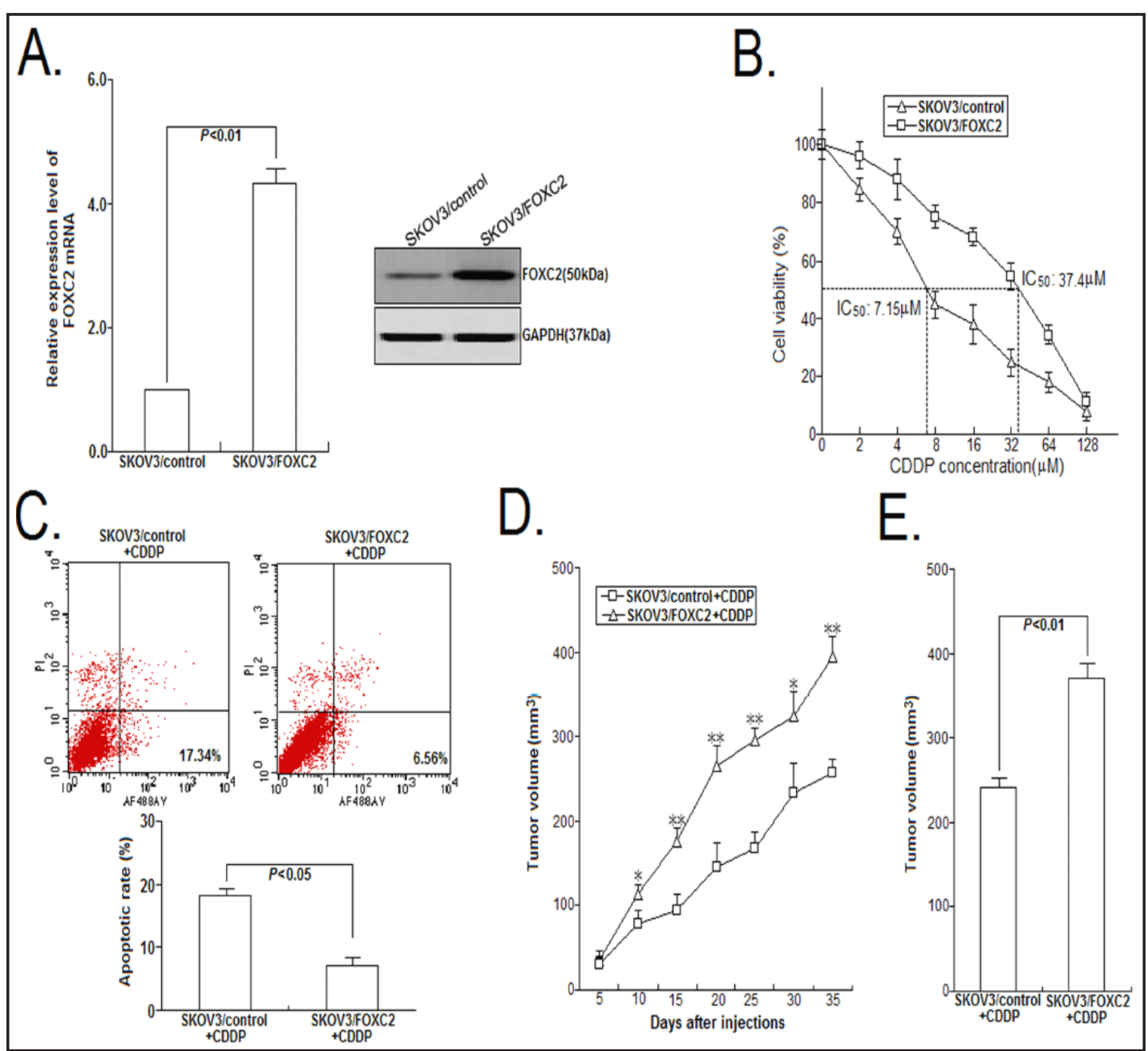

Fig. 3. Upregulation of FOXC2 significantly decreases the sensitivity of SKOV3 cells to CDDP in vitro and in vivo. (A) qRT-PCR and Western blot detection of FOXC2 mRNA and protein expression in SKOV3/FOXC2 and SKOV3/control cells, respectively. GAPDH serves as an internal control. (B) MTT analysis of the effects of FOXC2 upregulation on the IC $_{50}$ values of CDDP in SKOV3 cells. (C) Flow cytometric detection of apoptosis in SKOV3/FOXC2 or SKOV3/control cells with CDDP treatment (2.5 $\mu \mathrm{M})$. (D) The growth in mice of tumors developed from SKOV3/ control or SKOV3/FOXC2 cells with CDDP treatment. The inoculation was performed in two groups ( $n=10 /$ group). (E) After 35 days, the average tumor volume of tumors developed from SKOV3/FOXC2 cells with CDDP treatment was significantly bigger than that of tumors developed from SKOV3/control cells with CDDP treatment. All values represent the average of three independent experiments (mean $\pm \mathrm{SD}$ ). $* P<0.05$ and ${ }^{* *} P<0.01$, compared with the control.

$\left(394.2 \mathrm{~mm}^{3}\right.$ ) was significantly larger than that of tumors formed form SKOV3/control cells $\left(256.8 \mathrm{~mm}^{3}\right)(P<0.001$; Fig. 3E). Therefore, upregulation of FOXC2 significantly decreases the in vitro and in vivo sensitivity of CDDP-sensitive ovarian cancer cells to CDDP.

\section{Activation of Akt and MAPK signaling pathways are involved in FOXC2-promoting CDDP} resistance of ovarian cancer cells

Next, we will further investigate the molecular mechanisms involved in FOXC2promoting CDDP resistance of ovarian cancer cells. Previously, FOXC2 has been reported to activate the MAPK and AKT signaling pathways in other tumor cells. Here, it was observed that knockdown of FOXC2 could significantly induce the increased expression levels of p-Akt and p-ERK proteins in SKOV3/CDDP cells $(P<0.01)$, but the expression levels of 


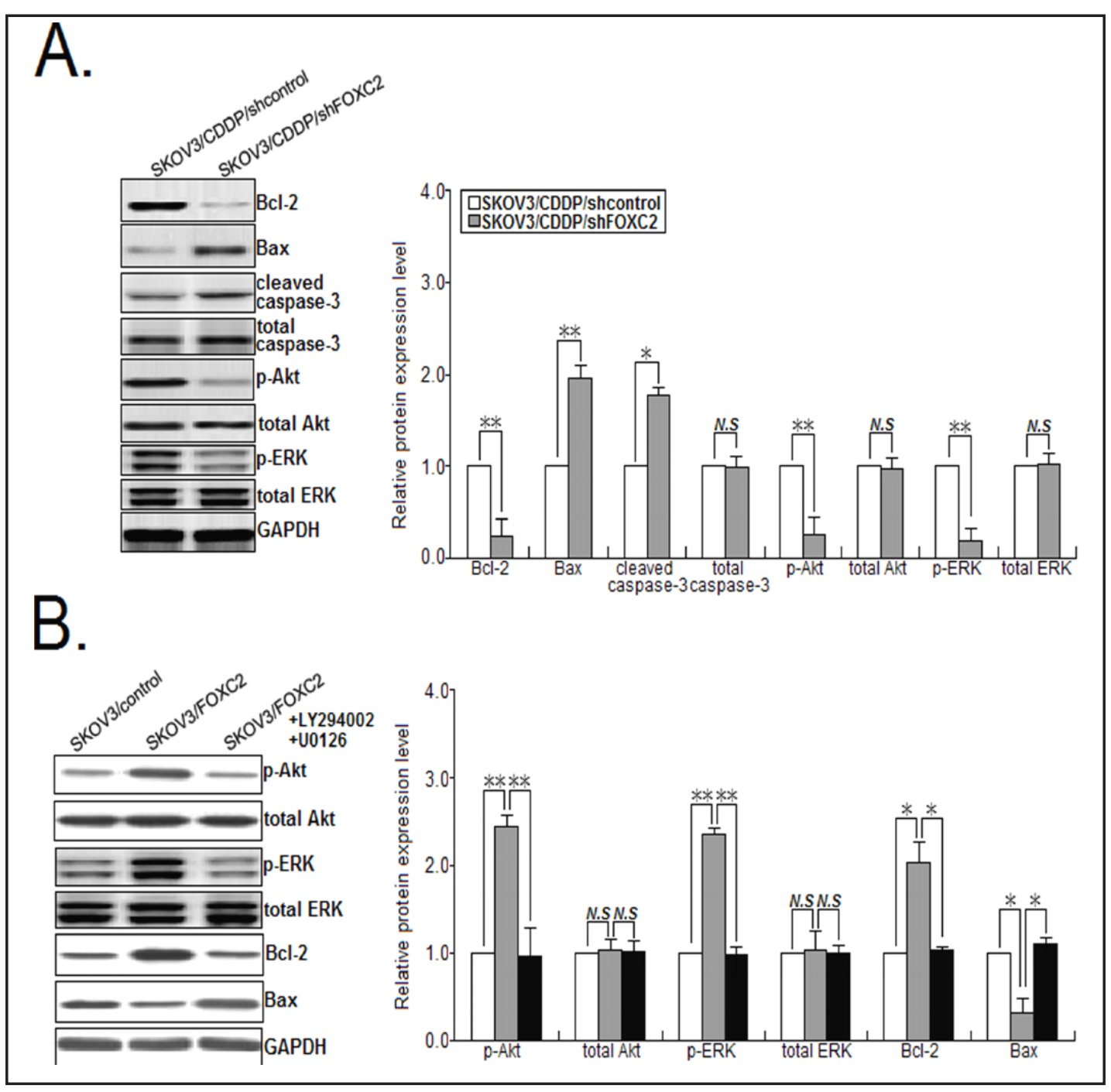

Fig. 4. The effect of FOXC2 expression on the expression of Akt or MAPK signaling-related proteins in ovarian cancer cells. (A) Western blot detection of the expression of Bcl-2, Bax, cleaved caspase-3, total caspase-3, p-Akt, total Akt, p-ERK and total ERK proteins in SKOV3/CDDP/shFOXC2 or SKOV3/CDDP/shcontrol cells, respectively. (B) Western blot detection of the expression of p-Akt, total Akt, p-ERK, total ERK, Bcl-2 and Bax proteins in SKOV3/control and SKOV3/FOXC2 cells or SKOV3/FOXC2 cells treated with LY294002 (8.0 $\mu$ M) plus U0126 $(10.0 \mu \mathrm{M})$, respectively. GAPDH serves as an internal control. All values represent the average of three independent experiments (mean $\pm \mathrm{SD}$ ). ${ }^{*} P<0.05$, ${ }^{* *} P<0.01$ and $N S, P>0.05$, compared with the control.

total Akt and ERK proteins showed no obvious changes in SKOV3/CDDP/shFOXC2 cells, in comparison with SKOV3/CDDP/shcontrol cells (Fig. 4A). Also, the decreased expression of Bcl-2 protein and the increased expression of Bax and cleaved caspase-3 proteins could be observed in SKOV3/CDDP/shFOXC2 cells, but the expression of total caspase-3 protein showed no obvious changes in SKOV3/CDDP/shFOXC2 cells, in comparison with SKOV3/ CDDP/shcontrol cells (Fig. 4A). To further confirm these results, we detected the expression of those above proteins in FOXC2-upregulated ovarian cancer cells (Fig. 4B). It was found that upregulation of FOXC2 could lead to the increased expression levels of p-Akt, p-ERK and Bcl-2 proteins and the decreased expression levels of Bax protein in SKOV3 cells, but the expression levels of total Akt and ERK proteins showed no obvious changes in SKOV3/ 
FOXC2 cells, in comparison with SKOV3/control cells. Importantly, treatment with Akt inhibitor (LY294002, 8.0 $\mu \mathrm{M}$ ) plus MAPK/ERK kinase inhibitor (U0126, $10.0 \mu \mathrm{M}$ ) could reverse the changes of p-Akt, p-ERK, Bcl-2 and Bax proteins in SKOV3/FOXC2 cells (Fig. 4B), in comparison with SKOV3/control cells. These data suggest that FOXC2 promotes the CDDP resistance in ovarian cancer cells via reducing apoptosis by activation of the Akt and MAPK signaling pathways.

\section{Discussion}

In the present study, we first showed that the expression levels of FOXC2 mRNA and protein were significantly upregulated in CDDP-resistant ovarian cancer tissues and cell lines. Knockdown of FOXC2 could reverse the CDDP resistance of SKOV3/CDDP cells, while upregulation of FOXC2 could decrease the sensitivity of SKOV3 cells to CDDP. Furthermore, FOXC2 could reduce the CDDP-induced apoptosis in ovarian cancer cells via activation of Akt and MAPK signaling pathways. To the best of my knowledge, this is the first report that FOXC2 plays a role in the development of CDDP resistance in human ovarian cancer cells.

The FOX proteins are transcription factors, which play critical roles in a variety of human physiological and pathological processes from development and organogenesis to regulation of metabolism, lymphatic vascular remodeling and tumor angiogenesis [16-18]. To date, human FOX gene family consists of at least 43 members, including FOXA1-3, FOXB1, FOXC1-2, FOXD1-6, etc [19]. Recently, FOX genes have been reported to be dysregulated in many human cancers and play different roles either as an oncogene or tumor suppressor in various tumor cells. Also, the polymorphisms of FOX genes was found to be correlated with an increased risk of human cancers and some FOX genes can be post-transcriptionally regulated by microRNAs [20-22]. The correlations of FOX proteins with ovarian cancer are increasingly reported. For example, Choi et al. showed that FOXP1 functions as an oncogene by promoting cancer stem cell-like characteristics in ovarian cancer cells [23]. Gao and his colleagues investigated the role of forkhead box Q1 transcription factor in ovarian epithelial carcinomas, and showed that FOXQ1 expression was essential to maintain cell proliferation, motility/invasion, and epithelial-mesenchymal transition phenotypes in ovarian cancer cells [24]. A study from Wen et al. suggest that overexpression of FOXM1 predicts poor prognosis and promotes cancer cell proliferation, migration and invasion in epithelial ovarian cancer [25]. Meanwhile, Lok and his colleagues found that over-expression of FOXM1 might stem from the constitutively active ERK which confers the metastatic capabilities to ovarian cancer cells [26]. FOXC2 is another important member of FOX gene family that has been reported in many cancers. The overexpression of FOXC2 were found be correlated with poor prognosis of tumor patients and promotes multiple malignant phenotypes of tumor cells, including proliferation, EMT, invasion and stem cell properties [27-30]. Importantly, the correlations between FOXC2 and tumor chemoresistance are also reported. Zhou et al. showed that FOXC2 could promote chemoresistance in nasopharyngeal carcinomas via induction of epithelial mesenchymal transition [31]. Also, Yang et al. reported that downregulation of Foxc2 could enhance apoptosis induced by 5 -fluorouracil in colorectal cancer [32]. Although overexpressed FOXC2 is reported to enhance the epithelial-to-mesenchymal transition and invasion of ovarian cancer cells, the roles of FOXC2 in the development of CDDP resistance in ovarian cancer cells is unclear and needs to be further investigated.

In this study, we first detected the expression of FOXC2 mRNA in ovarian cancer tissues from patients who received CDDP-based chemotherapy, and found that the relative expression of FOXC2 mRNA in CDDP-resistant ovarian cancer tissues was significantly higher than that in CDDP-sensitive tissues. Next, we detected the expression of FOXC2 mRNA and protein in CDDP-resistant ovarian cancer cell line SKOV3/CDDP and its parental cell line (SKOV3), and showed that the expression levels of FOXC2 both mRNA and protein in SKOV3/CDDP cell lines were significantly higher than those in SKOV3 cell line. These results implied that upregulation of FOXC2 might mediate the CDDP resistance of ovarian cancer

\section{KARGER}




\section{Cellular Physiology Cell Physiol Biochem 2016;39:242-252 \begin{tabular}{l|l} 
and Biochemistry 10.1159/000445620 & $\begin{array}{l}\text { DO } 2016 \text { The Author(s). Published by S. Karger AG, Basel } \\
\text { www.karger.com/cpb }\end{array}$ \\
\hline
\end{tabular} \\ Li et al.: FOXC2 and CDDP Resistance of Ovarian Cancer Cells}

cells. To testify this, we will perform gain-and loss-of-function assays. First, we employed RNA interfering technology to knockdown the expression of endogenous FOXC2 in SKOV3/ CDDP cells, and showed that knockdown of FOXC2 could induce the increased sensitivity of SKOV3/CDDP cells to CDDP both in vitro and in vivo by enhancing the CDDP-inducing apoptosis. Second, we employed gene overexpression technology to upregulate FOXC2 in SKOV3 cells. Results indicated that upregulation of FOXC2 could induce the decreased sensitivity of SKOV3 cells to CDDP both in vitro and in vivo by reducing the CDDP-inducing apoptosis. Followingly, the underlying molecular mechanisms involved in FOXC2-mediated CDDP resistance in ovarian cancer cells were further investigated. Previously, FOXC2 has been reported to promote colorectal cancer proliferation through inhibition of FOXO3a and activation of MAPK and AKT signaling pathways [33]. Also, FOXC2 enhanced AKT activity with subsequent GSK-3 $\beta$ phosphorylation and Snail stabilization, and then induced EMT and promoted tumor invasion and metastasis in colorectal cancer [34]. Yang et al. showed that downregulation of FOXC2 could enhance 5-fluorouracil-inducing apoptosis through activation of MAPK and AKT pathways in colorectal cancer [32]. However, whether FOXC2 promotes the chemoresistance of ovarian cancer cells to CDDP by activation of Akt and MAPK signaling pathways is unknown. Here, we found that knockdown of FOXC2 could lead to the decreased expression levels of p-Akt and p-ERK proteins but no changes in the expression of total Akt and ERK proteins, and then the decreased ratio of Bcl-2/Bax with subsequent enhancement of cleaved caspase-3 protein in SKOV3/CDDP cells. Meanwhile, upregulation of FOXC2 could lead to the increased expression levels of p-Akt and p-ERK proteins, and then the increased ratio of Bcl-2/Bax in SKOV3 cells. Importantly, treatment with Akt inhibitor plus MAPK/ERK kinase inhibitor could reverse the FOXC2 upregulation-induced changes of Bcl-2 and Bax proteins in SKOV3/FOXC2 cells. Thus, the regulation of Bcl-2, Bax and caspase- 3 by FOXC2 may result from activation of the Akt and MAPK signaling pathways. However, the possible molecular mechanism involved in FOXC2-mediated activation of the Akt and MAPK pathways needs to be further investigated. Additionally, it has been reported that ovarian cancer stem cells displayed higher chemoresistance to cisplatin or paclitaxel compared to adherent cells [35]. Thus, whether FOXC2 promotes CDDP resistance in ovarian cancer by regulating ovarian cancer stem cell properties will be determined in future.

In conclusion, FOXC2 is significantly upregulated in CDDP-resistant ovarian cancer tissues and cell lines. FOXC2 promotes the CDDP resistance of ovarian cancer cells via reduction of CDDP-inducing apoptosis by activating Akt and MAPK signaling pathways. Therefore, FOXC2 may be a potential molecular target for the treatment of CDDP-resistant ovarian cancer patients.

\section{Acknowledgements}

Thanks to every one of the Department of Obstetrics and Gynecology, Nanjing Maternity and Child Health Care Hospital to Nanjing Medical University, for their sincere help and technique support.

\section{Disclosure Statement}

No potential conflicts of interest were disclosed.

\section{References}

1 Siegel R, Naishadham D, Jemal A: Cancer statistics, 2013. CA Cancer J Clin 2013;63:11-30.

2 Song N, Liu H, Ma X, Zhang S: Placental growth factor promotes metastases of ovarian cancer through MiR543-regulated MMP7. Cell Physiol Biochem 2015;37:1104-1112.

\section{KARGER}




\section{Cellular Physiology Cell Physiol Biochem 2016;39:242-252 \begin{tabular}{c|c|c|} 
DOI: 10.1159/000445620 & $\begin{array}{l}\text { O 2016 The Author(s). Published by S. Karger AG, Basel } \\
\text { wwww.karger.com/cpb }\end{array}$ \\
and Biochemistry & Published online: June 24,2016
\end{tabular} \\ Li et al.: FOXC2 and CDDP Resistance of Ovarian Cancer Cells}

3 Chen Q, Qin R, Fang Y, Li H: Berberine Sensitizes Human Ovarian Cancer Cells to Cisplatin Through miR-93/ PTEN/Akt Signaling Pathway. Cell Physiol Biochem 2015;36:956-965.

4 Teng Y, Zhao L, Zhang Y, Chen W, Li X: Id-1, a protein repressed by miR-29b, facilitates the TGF $\beta 1$-induced epithelial-mesenchymal transition in human ovarian cancer cells. Cell Physiol Biochem 2014;33:717-730.

5 Ge T, Yin M, Yang M, Liu T, Lou G: MicroRNA-302b suppresses human epithelial ovarian cancer cell growth by targeting RUNX1. Cell Physiol Biochem 2014;34:2209-2220.

6 Agarwal R, Kaye SB: Ovarian cancer: strategies for over $\urcorner$ coming resistance to chemotherapy. Nat Rev Cancer 2003; 3:502-516.

7 Katoh M, Katoh M: Human FOX gene family (Review). Int J Oncol 2004;25:1495-500.

8 Sano H1, Leboeuf JP, Novitskiy SV, Seo S, Zaja-Milatovic S, Dikov MM, Kume T: The Foxc2 transcription factor regulates tumor angiogenesis. Biochem Biophys Res Commun 2010;392:201-206.

9 Mani SA, Yang J, Brooks M, Schwaninger G, Zhou A, Miura N, Kutok JL, Hartwell K, Richardson AL, Weinberg RA: Mesenchyme Forkhead 1 (FOXC2) plays a key role in metastasis and is associated with aggressive basal-like breast cancers. Proc Natl Acad Sci U S A 2007;104:10069-10074.

10 Wang YW, Yin CL, Zhang HY, Hao J, Yang YY, Liao H, Jiao BH: High expression of forkhead box protein C2 is related to poor prognosis in human gliomas. Asian Pac J Cancer Prev2014;15:10621-10625.

11 Nishida N, Mimori K, Yokobori T, Sudo T, Tanaka F, Shibata K, Ishii H, Doki Y, Mori M: FOXC2 is a novel prognostic factor in human esophageal squamous cell carcinoma. Ann Surg Oncol 2011;18:535-542.

12 Zhu JL, Song YX, Wang ZN, Gao P, Wang MX, Dong YL, Xing CZ, Xu HM: The clinical significance of mesenchyme forkhead 1 (FoxC2) in gastric carcinoma. Histopathology 2013;62:1038-1048.

13 Jiang W, Pang XG, Wang Q Shen YX, Chen XK, Xi JJ: Prognostic role of Twist, Slug, and Foxc2 expression in stage I non-small-cell lung cancer after curative resection. Clin Lung Cancer 2012;13:280-287.

14 Liu B, Han SM, Tang XY, Han L, Li CZ: Overexpressed FOXC2 in ovarian cancer enhances the epithelial-tomesenchymal transition and invasion of ovarian cancer cells. Oncol Rep 2014;31:2545-2554.

15 Luan QC, Sun YR, Han P, Chen Y: Ad-PUMA sensitizes ovarian cancer cells to chemotherapeutic agents. Eur Rev Med Pharmacol Sci 2015;19:4525-4532.

16 Kume T, Deng K, Hogan BL: Murine forkhead/winged helix genes Foxc1 (Mf1) and Foxc2 (Mfh1) are required for the early organogenesis of the kidney and urinary tract. Development 2000;127:1387-1395.

17 Li Y, Cai H, Fang W, Meng Q, Li J, Deng M, Long X: Fibroblast growth factor 2 involved in the pathogenesis of synovial chondromatosis of temporomandibular joint. J Oral Pathol Med 2014;43:388-394.

18 Kanady JD, Munger SJ, Witte MH, Simon AM: Combining Foxc2 and Connexin37 deletions in mice leads to severe defects in lymphatic vascular growth and remodeling. Dev Biol 2015;405:33-46.

19 Jackson BC, Carpenter C, Nebert DW, Vasiliou V: Update of human and mouse forkhead box (FOX) gene families. Hum Genomics 2010;4:345-352.

20 Wang Y, Zhou L, Chen J, Li J, He L, Wu P, Wang M, Tong N, Zhang Z, Fang Y: Association of the 3'UTR FOXO3a polymorphism rs4946936 with an increased risk of childhood acute lymphoblastic leukemia in a Chinese population. Cell Physiol Biochem 2014;34:325-332.

21 Song W, Li Q, Wang L, Wang L: Modulation of FoxO1 expression by miR-21 to promote growth of pancreatic ductal adenocarcinoma. Cell Physiol Biochem 2015;3:184-190.

22 Xu K, Liu X, Mao X, Xue L, Wang R, Chen L, Chu X: MicroRNA-149 suppresses colorectal cancer cell migration and invasion by directly targeting forkhead box transcription factor FOXM1. Cell Physiol Biochem 2015;35:499-515.

23 Choi EJ, Seo EJ, Kim DK, Lee SI, Kwon YW, Jang IH, Kim KH, Suh DS, Kim JH: FOXP1 functions as an oncogene in promoting cancer stem cell-like characteristics in ovarian cancer cells. Oncotarget 2016; 7:3506-3519.

24 Gao M, Shih IeM, Wang TL: The role of forkhead box Q1 transcription factor in ovarian epithelial carcinomas. Int J Mol Sci 2012;13:13881-13893.

25 Wen N, Wang Y, Wen L, Zhao SH, Ai ZH, Wang Y, Wu B, Lu HX, Yang H, Liu WC, Li Y: Overexpression of FOXM1 predicts poor prognosis and promotes cancer cell proliferation, migration and invasion in epithelial ovarian cancer. J Transl Med 2014;12:134.

26 Lok GT, Chan DW, Liu VW, Hui WW, Leung TH, Yao KM, Ngan HY: Aberrant activation of ERK/FOXM1 signaling cascade triggers the cell migration/invasion in ovarian cancer cells. PLoS One 2011;6:e23790. 


\section{Cellular Physiology Cell Physiol Biochem 2016;39:242-252 \begin{tabular}{l|l|l} 
DOI: 10.1159/000445620 & $\begin{array}{l}\text { O 2016 The Author(s). Published by S. Karger AG, Basel } \\
\text { www.karger.com/cpb }\end{array}$
\end{tabular} \\ Li et al.: FOXC2 and CDDP Resistance of Ovarian Cancer Cells}

27 Imayama N, Yamada S, Yanamoto S, Naruse T, Matsushita Y, Takahashi H, Seki S, Fujita S, Ikeda T, Umeda M: FOXC2 expression is associated with tumor proliferation and invasion potential in oral tongue squamous cell carcinoma. Pathol Oncol Res 2015;21:783-791.

28 Cui YM, Jiao HL, Ye YP, Chen CM, Wang JX, Tang N, Li TT, Lin J, Qi L, Wu P, Wang SY, He MR, Liang L, Bian XW, Liao WT, Ding YQ: FOXC2 promotes colorectal cancer metastasis by directly targeting MET. Oncogene. 2015;34:4379-4390.

29 Watanabe A, Suzuki H, Yokobori T, Altan B, Kubo N, Araki K, Wada S, Mochida Y, Sasaki S, Kashiwabara K, Hosouchi Y, Kuwano H: Forkhead box protein C2 contributes to invasion and metastasis of extrahepatic cholangiocarcinoma, resulting in a poor prognosis. Cancer Sci 2013;104:1427-1432.

30 Hollier BG, Tinnirello AA, Werden SJ, Evans KW, Taube JH, Sarkar TR, Sphyris N, Shariati M, Kumar SV, Battula VL, Herschkowitz JI, Guerra R, Chang JT, Miura N, Rosen JM, Mani SA: FOXC2 expression links epithelial-mesenchymal transition and stem cell properties in breast cancer. Cancer Res 2013;73:19811992.

31 Zhou Z, Zhang L, Xie B, Wang X, Yang X, Ding N, Zhang J, Liu Q Tan G, Feng D, Sun LQ: FOXC2 promotes chemoresistance in nasopharyngeal carcinomas via induction of epithelial mesenchymal transition. Cancer Lett 2015;363:137-145.

32 Yang C, Cui X, Dai X, Liao W: Downregulation of Foxc2 enhances apoptosis induced by 5-fluorouracil through activation of MAPK and AKT pathways in colorectal cancer. Oncol Lett 2016;11:1549-1554.

33 Cui YM, Jiang D, Zhang SH, Wu P, Ye YP, Chen CM, Tang N, Liang L, Li TT, Qi L, Wang SY, Jiao HL, Lin J, Ding YQ Liao WT: FOXC2 promotes colorectal cancer proliferation through inhibition of FOXO3a and activation of MAPK and AKT signaling pathways. Cancer Lett 2014;353:87-94.

34 Li Q Wu J, Wei P, Xu Y, Zhuo C, Wang Y, Li D, Cai S: Overexpression of forkhead Box C2 promotes tumor metastasis and indicates poor prognosis in colon cancer via regulating epithelial-mesenchymal transition. Am J Cancer Res 2015;5:2022-2034.

35 He QZ, Luo XZ, Wang K, Zhou Q Ao H, Yang Y, Li SX, Li Y, Zhu HT, Duan T: Isolation and characterization of cancer stem cells from high-grade serous ovarian carcinomas. Cell Physiol Biochem 2014;33:173-184. 